\title{
Emphysematous Pyelonephritis in a Patient With Hyperosmolar Hyperglycemic Syndrome
}

\author{
Hidekatsu Yanaia, b $^{\text {, }}$ Keisuke Sekine ${ }^{a}$
}

\begin{abstract}
A 61-year-old woman presented with abdominal and back pain, and disturbed consciousness. Laboratory data showed hyperglycemia (plasma glucose $448 \mathrm{mg} / \mathrm{dl}$ ), leukocytosis, thrombocytopenia, renal failure, and extremely increased serum C-reactive protein level. Urinalysis revealed no ketone bodies, and numerous bacteria and leukocytes. These symptoms and data suggested the presence of diabetic hyperosmolar hyperglycemic syndrome and urinary tract infection. Computed tomography (CT) of the abdomen showed the presence of gas in the renal area with extensive renal parenchymal destruction, indicating the diagnosis of emphysematous pyelonephritis, which is a rare but severe disease with a morality rate that can be as high as $70 \%$. Early diagnosis by abdominal CT and early intensive therapy using intensive insulin therapy, antibiotics, and selective nephrectomy saved her life.
\end{abstract}

Keywords: Computed Tomography; Emphysematous pyelonephritis; Hyperosmolar hyperglycemic syndrome

\section{Introduction}

Emphysematous pyelonephritis (EPN) is a severe acute necrotizing infection of the renal parenchyma and perirenal tissue, characterized by gas formation. EPN is a rare but severe disease with a morality rate that can be as high as $70 \%$ [1].

Manuscript accepted for publication January 4, 2011

${ }^{\mathrm{a}}$ Department of Internal Medicine, National Center for Global Health and Medicine, Kohnodai Hospital, Chiba, Japan

${ }^{\mathrm{b}}$ Corresponding author: Hidekatsu Yanai, Department of Internal Medicine, National Center for Global Health and Medicine, Kohnodai Hospital, 1-7-1 Kohnodai, Ichikawa, Chiba 272-8516, Japan. Email: dyanai@hospk.ncgm.go.jp

doi:10.4021/jmc121w
EPN occurs invariably in diabetic patients [1]. Prompt diagnosis and early treatment are crucial for the recovery from EPN, as this disease progresses quickly. However, the diagnosis is sometimes made after poor response to conventional antibiotic treatment [1].

We report a case with diabetic hyperosmolar hyperglycemic syndrome (HHS) complicated with EPN, who was successfully treated by early diagnosis and early intensive therapy using insulin, antibiotics, and nephrectomy.

\section{Case Report}

A 61-year-old woman was referred for hyperglycemia. She discontinued to receive the treatment for diabetes mellitus 3 years ago. She presented with abdominal and back pain for the previous 6 days, and her consciousness was disturbed. Her body temperature was $36.3{ }^{\circ} \mathrm{C}$ and blood pressure was $82 / 42 \mathrm{mmHg}$. Plasma glucose level $(448 \mathrm{mg} / \mathrm{dl})$ and hemoglobin A1C level (13\%; normal range, 4.3 - 5.8\%) were elevated. Laboratory data showed increased leukocyte counts $(25,000 / \mu \mathrm{l})$ and decreased platelet counts $(72,000 / \mu \mathrm{l})$. Serum levels of blood urea nitrogen ( $78 \mathrm{mg} / \mathrm{dl}$; normal range, 8 - 22 $\mathrm{mg} / \mathrm{dl})$, creatinine $(2.2 \mathrm{mg} / \mathrm{dl}$; normal range, $0.4-0.7 \mathrm{mg} / \mathrm{dl})$ and C-reactive protein $(38.9 \mathrm{mg} / \mathrm{dl}$; normal range, 0 - $0.3 \mathrm{mg} /$ dl) were remarkably elevated. Urinalysis revealed no ketone bodies, and numerous bacteria and leukocytes. These symptoms and data suggested the presence of HHS and urinary tract infection (UTI). Axial section (Fig. 1a) and coronal section (Fig. 1b) of computed tomography (CT) of the abdomen showed the presence of gas in the renal area with extensive renal parenchymal destruction, indicating the diagnosis of EPN. Cultures of blood and urine grew Escherichia coli. She was successfully treated by intensive insulin therapy, antibiotics and left nephrectomy.

\section{Discussion}

EPN has been defined as an acute, severe, necrotizing infection of the renal parenchyma and perirenal tissue, which results in the appearance of gas within the renal parenchy- 
(a)

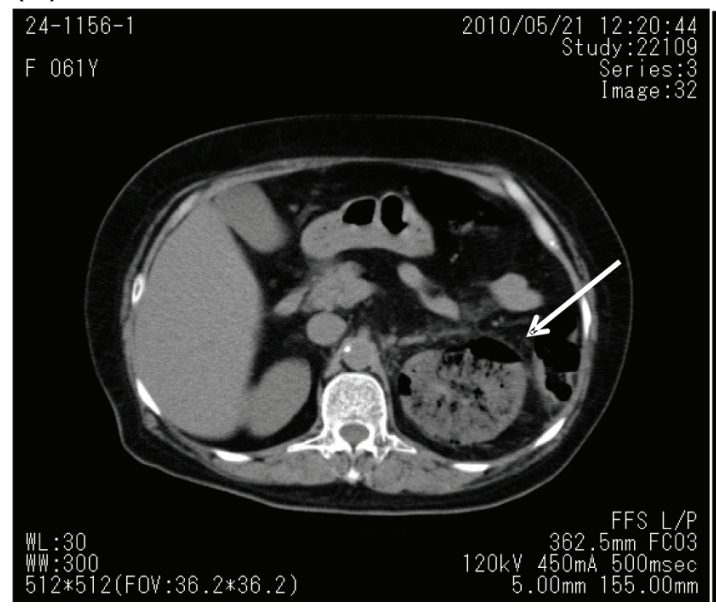

(b)

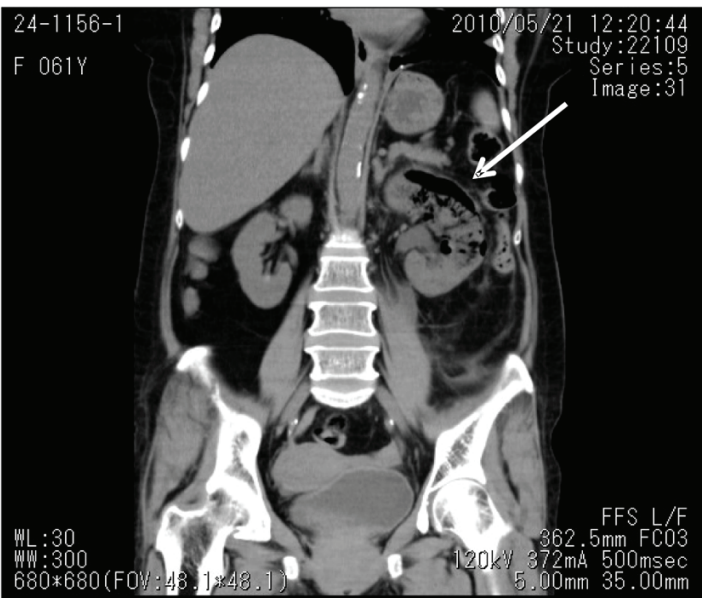

Figure 1. Axial (a) and coronal (b) section of computed tomographic image of emphysematous pyelonephritis (arrows).

ma, and EPN particularly occurs in diabetes [1]. The clinical features of EPN are indistinguishable from those of UTI. In this patient, the overwhelming hyperglycemic hyperosmolar state makes the diagnosis for EPN difficult. A high index of suspicion, especially in susceptible populations such as diabetic patients, is needed for early diagnosis. A delay in diagnosis leads to increased morbidity and mortality [1]. The best diagnostic modality has been reported to be CT $[2,3]$, which can also be used in patients who are suitable for CT-guided drainage. In fact, abdominal CT was very useful for the diagnosis of EPN in our patient. High-risk patients with EPN have been considered to be patients with shock, thrombocytopenia, acute renal failure and disturbed consciousness, all of which were observed in our patient [4]. Furthermore, HHS has also been identified as a disease with a high mortality rate [5]. We believe that early diagnosis by abdominal CT and early intensive therapy using insulin, antibiotics, and selective nephrectomy saved her life.

\section{References}

1. Mokabberi R, Ravakhah K. Emphysematous urinary tract infections: diagnosis, treatment and survival (case review series). Am J Med Sci 2007;333(2):111-116.

2. Koh KB, Lam HS, Lee SH. Emphysematous pyelonephritis: drainage or nephrectomy? $\mathrm{Br} \mathrm{J}$ Urol 1993;71(5):609-611.

3. Pontin AR, Barnes RD, Joffe J, Kahn D. Emphysematous pyelonephritis in diabetic patients. Br J Urol 1995;75(1):71-74.

4. Huang JJ, Tseng CC. Emphysematous pyelonephritis: clinicoradiological classification, management, prognosis, and pathogenesis. Arch Intern Med 2000;160(6):797805.

5. Small M, Alzaid A, MacCuish AC. Diabetic hyperosmolar non-ketotic decompensation. Q J Med 1988;66(251):251-257. 